\title{
WORLD UNIVERSITY SERVICE (WUS) ENTRAIDE UNIVERSITAIRE MONDIALE (EUM) SERVICIO UNIVERSITARIO MUNDIAL (SUM)
}

5 , chemin des Iris 1216 Geneva (Switzerland)

Tel: (22) 7988711

Fax: (22) 7980829

Telex: 27273 wus ch

Cables: interstud geneva

President: Caleb Fundanga

Secretary-General: Nigel Hartley

A non-governmental organization and network of forty-five national committees, three corresponding committees and ten contact groups in Africa, Asia, Europe and North America. WUS main activities include: community development and social action programmes; education and training programmes for refugees, returnees and victims of persecution from South Africa and Namibia, Latin America/Caribbean and for Palestinians; defense of humanrights; women's programmes; and academic solidarity and cooperation.

Principal publications: WUS and Human
Rights; WUS News; Report on Academic Freedom (annually).

L'Entraide universitaire mondiale (EUM) est une organisation non-gouvernementale constituée par un réseau de quarante-cinq comités nationaux, trois comités correspondants et dix groupes de liaison en Afrique, Asie, Europe et Amérique du Nord. Les activités principales de l'EUM comprennent: le développement de la communauté et des programmes d'action sociale; des programmes d'enseignement et de formation pour les réfugiés et victimes des persécutions en Afrique du Sud, Namibie, Amérique Latine/Caraibes et en Palestine; la défense des droits de l' homme; des programmes pour les femmes; enfin, des programmes de solidarité et de coopération universitaires.

Publications principales: "WUS \& Human Rights"; "WUS New's"; "Report on Academic Freedom" (annuel). 